\section{Research Square}

Preprints are preliminary reports that have not undergone peer review.

They should not be considered conclusive, used to inform clinical practice, or referenced by the media as validated information.

\title{
Six Year Trend Analysis of Malaria Prevalence at University of Gondar Specialized Referral Hospital, Northwest Ethiopia, from 2014-2019
}

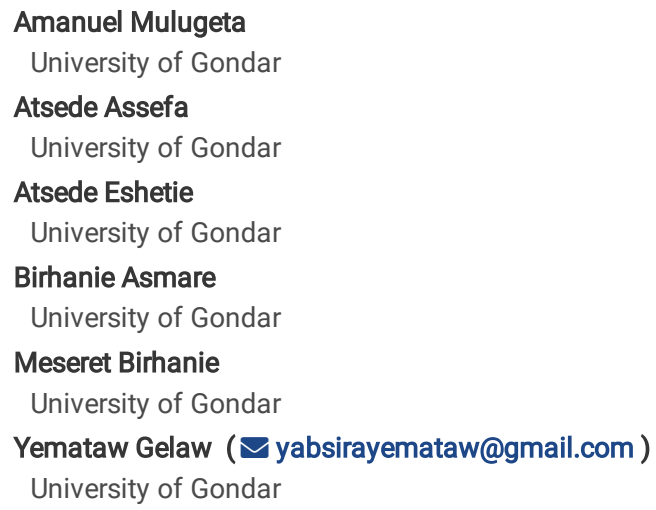

Research

Keywords: Malaria, P.falcifarum, Prevalence, P.vivax Trend

Posted Date: July 1st, 2021

DOI: https://doi.org/10.21203/rs.3.rs-656381/v1

License: () (7) This work is licensed under a Creative Commons Attribution 4.0 International License. Read Full License 


\section{Abstract}

Background: Malaria is disease caused by plasmodium species and transmitted by the bite of female anopheles mosquito. Globally, it is still the major public health problem that causes morbidity and mortality especially in tropical and sub-tropical African countries including Ethiopia. The objective of this study was to assess the Six year trend of malaria at University of Gondar Specialized Referral Hospital, northwest Ethiopia, from 2014-2019

Method: A retrospective study was conducted by reviewing the laboratory registration logbook at University of Gondar Specialized Referral Hospital by using data extraction tool. The data was analysized by SPSS version 20. Descriptive statistics was used to summarize the socio-demographic characteristics of study participants and presented by graphs, tables and texts. The binary logistic regression was also used test the association of trend of malaria and different factors like sex, age, year, and season.

Result: From a total 17,500 malaria suspected patients, 1341 (7.7\%) were confirmed for malaria and (47.2\%), (45.6\%) and (7.2\%) were P.vivax, P. falciparum and mixed infection, respectively. P.vivax was the predominant species in the first three years and P. falciparum became the predominant species in the last three years. The trend prevalence was showed a fluctuated significantly decrement. It decreased by $68 \%, 60 \%$ and $69 \%$ in the year of 2017,2018 and 2019 compare to 2014 , respectively. The positivity rate were significantly higher in the males (1.41 times higher) than females. It was also significantly higher in lower age groups compare to the older age groups (> 54 years old). It was 1.60, 1.64, 2.45 and 1.82 times higher in the age group of < 5, 5-14, 15-24 and 25-54 years old, respectively.

Conclusion: There has been a significant declining trend of malaria in the study area. However, it is still a major health problem and requires extra efforts for further reduction. Even, autumn was the season when the highest peak of malaria cases in was observed, there was high fluctuation from year to year. Therefore, control activities should be continued and strengthened manner in the study area considering these variability.

\section{Background}

Malaria is one of the protozoan blood parasite globally that cause morbidity and mortality(1). It has been a major public health problem throughout human history, particularly in the tropical and subtropical parts of the world. The problem of malaria is very severe in Ethiopia where it has been the major cause of illness and death for many years. According to records from the Ethiopian Federal Ministry of Health, $75 \%$ of the country is malarious with about $68 \%$ of the total population living in areas at risk of malaria (2).

Due to the unstable nature of malaria transmission, malaria has been the leading cause of morbidity and mortality in Ethiopia. The two peak seasonal transmissions of malaria in Ethiopia are the months of September to December and March to May $(3,4)$. Studies have shown that the Plasmodium species compositions and the number of malaria cases vary over time due to different factors, such as previous weather conditions or intervention measures (3). A health facility based retrospective studies in Ethiopia revealed decrement of Plasmodium species over period years $(5,6)$. Another trend studies showed fluctuation of malaria cases $(4,7,8)$.

Understanding how malaria varies in the community as a result of seasonal or year-to-year changes is essential for planning national malaria control programs (6). The analysis of relevant malaria data of health care system gives essential information to measure achievements of national malaria programs and identify malaria hot spots. It also gives important insight into the changing malaria situation, which might guide adjustments of malaria program activities and the prioritization of malaria research and the changing malaria situation requires an updating description of malaria trends $(9,10)$.

Population based studies regarding prevalence of malaria over a period of time that includes all age segments of the population, taking into account the base population (denominator), are scarce in Ethiopia in spite of the recognition of the disease burden for more than half of a century. Therefore, the objectives of this study were to analyze trends of malaria prevalence at University of Gondar Specialized Referral Hospital, northwest Ethiopia to identify trends of Plasmodium species over the time-period.

\section{Methods}

\section{Study area and study population}

The study was conducted at University of Gondar specialized referral hospital, Northwest Ethiopia located in Gondar town. Gondar is ancient city which is located $727 \mathrm{~km}$ away from Addis Ababa, the capital city of Ethiopia and 175km from Bahir Dar, the capital city of Amhara regional state. The town has latitude and longitude $12^{\circ} 361 \mathrm{~N} 37^{\circ} 281 \mathrm{E}$ with an elevation of 2133 meters above sea level. According to Central Statistical Agency of Ethiopia 2015 report, it has twelve sub city and 22 urban and 11 rural kebeles with a projected population of 323,900. The city has 8 public health centers and 1 public specialized comprehensive referral hospital (University of Gondar specialized referral hospital), more than 13 private clinics and 1 general hospital providing health services like diagnosis, treatment, prevention and control of diseases (11). The study population in this study was all malaria suspected patients (including both sexes and any age groups) who were visiting the health centre for the past 6 years (from 2014-2019).

\section{Study Design}

A retrospective study was conducted to determine the 6 years (2014-2019) trend of malaria by reviewing blood film malaria result at laboratory registration $\log$ book. 


\section{Data Collection}

Six years (2014-2019) data regarding malaria were extracted from University of Gondar referral Hospital laboratory registration log book by using data extraction sheet. The data collection sheet includes result of blood film (Negative and Positive), type of plasmodium species ( $p$. falciparum, p. vivax and mixed), year of examination, month of examination, season of examination, sex and age of the patient. Data on both negative and positive microscopically confirmed malaria suspected cases were included in the study. In Ethiopia, microscopy is the major diagnostic method for malaria. A well-prepared Gimsa stained blood film (both thick and thin smear) was used to diagnose malaria in the laboratory. Unfortunately, complete data regarding major interventions done against malaria and other environmental factors were not collected.

\section{Data Analysis And Interpretation}

The data were checked for completeness, cleaned, and sorted daily and entered into SPSS version 20 for analysis. Descriptive statistics were used to summarize the socio-demographic of study participants and the frequency of malaria on different independent variables and presented by tables and texts. Multivariable binary logistic regression analyses were performed to determine the association between the dependent (malaria prevalence and independent variables (age, sex, and year and season as categorical variable). The multivariable binary logistic regression model was analyzed with enter method and a $\mathrm{p}$ value $<0.05$ in the multivariable regression model was considered as statistically significant. The model fitness of the final multivariable logistic regression was checked using Hosmer and Lemeshow test.

\section{Result}

\section{Characteristics of study participants requested for malaria diagnosis}

During 2014 to 2019, a total of 17,500 blood films (in average 2917 blood films per year) were examined microscopically for malaria diagnosis. More than half of the cases were males, 9542 (55.5\%) and this was more or less consistent throughout the six years. In the six trends, the most malaria suspected and examined cases were in the age group of 25-54 (7040 (40.2\%)) followed by age group of 15-24 (5540 (31.7\%)) and the lowest suspected case was examined in the older age groups (> 54 years old) (1485 (8.5)). The trend of suspected cases for malaria examination was high fluctuated. The highest blood film examination was performed in the year of 2015 (2789 (24.1\%)) followed by year of 2017 (3348 (19.1\%)) (Table 1).

Table 1

Socio-demographic characteristics of patients request for malaria examination at University of Gondar Specialized Referral Hospital from 2014 to 2019

\begin{tabular}{|c|c|c|c|c|c|c|c|c|}
\hline Socio-demographic variable & & Year & & & & & & \\
\hline \multirow[t]{3}{*}{ Sex $n(\%)$ from sex } & & 2014 & 2015 & 2016 & 2017 & 2018 & 2019 & Total \\
\hline & Female & $1072(47.4)$ & $1900(45.1)$ & $1258(45.1)$ & $1540(46.0)$ & $1314(42.0)$ & $874(49.4)$ & $7958(45.5)$ \\
\hline & Male & $1188(52.6)$ & $2310(54.9)$ & $1531(54.9)$ & $1808(54.0)$ & $1811(58.0)$ & $894(50.6)$ & $9542(55.5)$ \\
\hline \multirow[t]{5}{*}{ Age in year $n(\%)$ from age group } & $<5$ & $169(7.5)$ & $215(5.1)$ & $246(8.8)$ & $316(9.4)$ & $333(10.7)$ & $300(17.0)$ & $1579(9.0)$ \\
\hline & $5-14$ & $227(10)$ & $400(9.5)$ & $269(9.6)$ & 307 (9.2) & $317(10.1)$ & $336(19)$ & $1856(10.6)$ \\
\hline & $15-24$ & $753(33.3)$ & $1545(36.7)$ & $917(32.9)$ & $1015(30.3)$ & $900(28.8)$ & $410(23.2)$ & $5540(31.7)$ \\
\hline & $25-54$ & $931(41.2)$ & $1719(40.8)$ & $1106(39.7)$ & $1412(42.2)$ & $1274(40.8)$ & $598(33.8)$ & $7040(40.2)$ \\
\hline & $>54$ & $180(8)$ & $331(7.9)$ & $251(9)$ & $298(8.9)$ & $301(9.6)$ & $124(7)$ & $1485(8.5)$ \\
\hline$N$ (\%) from Total & & 2260 (12.9) & $4210(24.1)$ & 2789 (15.9) & 3348 (19.1) & $3125(17.9)$ & $1768(10.1)$ & $17500(100)$ \\
\hline
\end{tabular}

Among a total of 17,500 examined blood films, 1341 (7.7\%; $95 \% \mathrm{Cl} ; 7.3-8.1)$ were positive for plasmodium species during the six year period. There were significant fluctuations and reduction trends of overall malaria during the past 6 years, with a maximum of $11.2 \%$ and a minimum of $3.7 \%$ of cases in 2016 and 2019, respectively. P.vivax was the predominant plasmodium species. However, the proportion of the plasmodium species was significantly fluctuated in the six years period (chi squared $=62.58$, $P$ value $<0.001$ ). In the first 3 years P. vivax was the predominant plasmodium species and in the last 3 years P.falcifarum was the predominant malaria case. Moreover, mixed infection (P.vivax and P.falcifarum) showed a significant fluctuating increment trend in the area in the 6 years, with a maximum of $10.9 \%$ and a minimum of $3.6 \%$ of cases in 2017 and 3 in 2014, respectively (Table 2 and Fig. 1 ). 
Table 2

Annual trend of malaria prevalence and proportion of plasmodium species in each year among patients requested for malaria examination at University of Gondar Specialized Referral Hospital from 2014 to 2019

\begin{tabular}{|c|c|c|c|c|c|c|}
\hline \multicolumn{2}{|c|}{ Variable } & \multirow[t]{2}{*}{ Total No Patient N (\%) } & \multirow[t]{2}{*}{ Total Positivity rate $\mathrm{n}(\% ; 95 \% \mathrm{Cl})$} & \multicolumn{3}{|c|}{ Plasmodium species proportion $(n=1341)$} \\
\hline & & & & P.f n (\%) & P.v n (\%) & Mixed n (\%) \\
\hline \multirow[t]{6}{*}{ Year } & 2014 & 2260 & $237(10.5 ; 9.3-11.8)$ & $84(35.4)$ & $146(61.6)$ & $7(3.0)$ \\
\hline & 2015 & 4210 & $460(10.9 ; 10.0-11.9)$ & $190(41.3)$ & $232(50.4)$ & $38(8.3)$ \\
\hline & 2016 & 2789 & $311(11.2 ; 10.0-12.4)$ & 137(44.2) & $146(47.1)$ & $27(8.7)$ \\
\hline & 2017 & 3348 & $128(3.8 ; 3.2-4.5)$ & $76(59.4)$ & $38(29.7)$ & 14(10.9) \\
\hline & 2018 & 3125 & $139(4.4 ; 3.8-5.2)$ & $87(62.6)$ & $47(33.8)$ & $5(3.6)$ \\
\hline & 2019 & 1768 & $66(3.7 ; 2.9-4.7)$ & $37(55.2)$ & $24(35.8)$ & $6(9.0)$ \\
\hline Total & & 17500 & $1341(7.7 ; 7.3-8.1)$ & $611(45.6)$ & $633(47.2)$ & $97(7.2)$ \\
\hline
\end{tabular}

\section{Sex, Age And Seasonal Variations Of Malaria Prevalence}

Despite the apparent fluctuation of total malaria trends over 6 years in the study area, malaria cases occurred throughout the year. However, there was a significant variation between the two sexes and different age groups. The odds of malaria prevalence among the male was 1.41 (95\%Cl; $1.26-1.59$ ) times higher than females. The prevalence of malaria was also higher in lower age groups compare to the older age groups. The odds of malaria prevalence was 1.60 (95\% Cl; 1.14-2.23), 1.64 (95\% Cl; $1.20-2.26), 2.45$ (95\% Cl; $1.86-3.22)$ and 1.82 (95\%Cl; $1.39-2.40)$ in the age group of < 5 years, $5-14$ years, $15-24$ years and 25-54 years, respectively compare to age group of $>54$ years old. Controlling of the confounding factors sex and age, the prevalence of malaria also showed significant reduction in the last 3 years (2017-2019) compare to the first year (2014). The prevalence of malaria was decreased by $68 \%$ (95\% Cl; $60-75), 60 \%(95 \% \mathrm{Cl} ; 51-68)$ and $69 \%(95 \% \mathrm{Cl} ; 59-77)$ in the year of 2017, 2018 and 2019, respectively. Moreover, there was a significant seasonal variation in malaria cases. The highest peak of total malaria positivity rate was observed during autumn, (September, October and November; just after the main rainy season) and the minimum positivity rate was observed during winter (the dry season in the months of December, January and February) and showed significant variation. However, controlling of the sex and age group variation in the season, the highest peak of total malaria positivity rate was observed during summer (June, July and August; main rainy season). Moreover, the seasonal variation was not consistent and highly fluctuated in the six years. Even it was the season where the highest malaria case was reported in over all seasonal malaria prevalence, autumn was the season where lowest malaria case was report in 2014 and 2017 (Table 3, and Figs. 2 and 3). 
Table 3

Sex, age and seasonal variations of malaria prevalence and associated factors among patients requested for malaria examination at University of Gondar Specialized Referral Hospital from 2014 to 2019.

\begin{tabular}{|c|c|c|c|c|}
\hline \multirow[t]{2}{*}{ Variable } & & \multicolumn{2}{|c|}{ Smear microscopy result } & \multirow[t]{2}{*}{ AOR $(95 \% \mathrm{Cl})$} \\
\hline & & Negative n (\%) & Positive n (\%) & \\
\hline \multirow[t]{2}{*}{ Sex } & Female & $7,454(93.7)$ & $504(6.3)$ & 1 \\
\hline & Male & 8,705 (91.2) & $837(8.8)$ & $1.41(1.26-1.59)$ \\
\hline \multirow[t]{5}{*}{ Age } & $<5$ & $1486(94.1)$ & $93(5.9)$ & $1.60(1.14-2.23)$ \\
\hline & $5-14$ & $1736(93.5)$ & $120(6.5)$ & $1.64(1.20-2.26)$ \\
\hline & $15-24$ & $4,986(90)$ & $554(10.0)$ & $2.45(1.86-3.22)$ \\
\hline & $25-54$ & $6,526(92.7)$ & $514(7.3)$ & $1.82(1.39-2.40)$ \\
\hline & $>54$ & $1424(95.9)$ & $61(4.1)$ & 1 \\
\hline \multirow[t]{6}{*}{ Year } & 2014 & $2,023(89.5)$ & $237(10.5)$ & 1 \\
\hline & 2015 & $3,750(89.1)$ & $460(10.9)$ & $1.02(0.86-1.20)$ \\
\hline & 2016 & 2,478 (88.8) & $311(11.2)$ & $1.07(0.89-1.28)$ \\
\hline & 2017 & $3,220(96.2)$ & $128(3.8)$ & $0.32(0.25-0.40)$ \\
\hline & 2018 & $2,986(95.6)$ & $139(4.4)$ & $0.40(0.32-0.49)$ \\
\hline & 2019 & $1,702(96.3)$ & $66(3.7)$ & $0.31(0.23-0.41)$ \\
\hline \multirow[t]{4}{*}{ Season } & Autumn & 3859 (91.2) & $374(8.8)$ & 1 \\
\hline & Winter & 4002 (93.4) & $281(6.6)$ & $0.84(0.71-0.99)$ \\
\hline & Spring & $4503(92.5)$ & $366(7.5)$ & $1.01(0.86-1.18)$ \\
\hline & Summer & 3795 (92.2) & $320(7.8)$ & $1.32(1.12-1.55)$ \\
\hline
\end{tabular}

The highest prevalence of malaria was seen in the month of August (9.6\%) followed by September and November (9.3\%) whereas, the lowest prevalence was seen in the month of January (6.1\%). The proportion of plasmodium species were highly fluctuated throughout the 12 months. Plasmodium vivax was predominantly high in the winter months (December, January and February), spring months (March, April and May) and the two autumn months (September and November) whereas, plasmodium falciparum was predominantly high in the summer months (June, July and August) and one of the autumn month (October). The mixed infection was also shoed monthly fluctuation in which the highest peak was observed in the month of March and the lowest peak was observed in the month of December (Table 4).

Table 4

Monthly prevalence of malaria and proportion of plasmodium species among patients requested for malaria examination at University of Gondar Specialized Referral Hospital from 2014 to 2019

\begin{tabular}{|c|c|c|c|c|c|c|}
\hline \multirow[t]{2}{*}{ Variable } & & \multirow[t]{2}{*}{ Total No Patient N (\%) } & \multirow[t]{2}{*}{ Total Positivity rate $\mathrm{n}(\%)$} & \multicolumn{3}{|c|}{ Plasmodium species proportion $(n=1341)$} \\
\hline & & & & P.f n (\%) & P.v $n(\%)$ & Mixed n (\%) \\
\hline \multirow[t]{12}{*}{ Month } & December & 1199 & $79(6.6)$ & $36(45.6)$ & $40(50.6)$ & $3(3.8)$ \\
\hline & January & 1551 & $95(6.1)$ & $39(41.1)$ & $49(51.6)$ & $7(7.4)$ \\
\hline & February & 1533 & $107(7.0)$ & $39(36.4)$ & $59(55.1)$ & $9(8.4)$ \\
\hline & March & 1724 & $115(6.7)$ & $37(32.2)$ & $66(57.4)$ & $12(10.4)$ \\
\hline & April & 1633 & $123(7.5)$ & $57(46.3)$ & $58(47.2)$ & $8(6.5)$ \\
\hline & May & 1512 & $128(8.5)$ & $58(45.3)$ & $66(51.6)$ & $4(3.1)$ \\
\hline & June & 1061 & $72(6.8)$ & $47(65.3)$ & 19 (26.4) & $6(8.3)$ \\
\hline & July & 1422 & $92(6.5)$ & $53(57.6)$ & $33(35.9)$ & $6(6.5)$ \\
\hline & August & 1632 & $156(9.6)$ & $84(53.8)$ & $63(40.4)$ & $9(5.8)$ \\
\hline & September & 1226 & $113(9.3)$ & $43(38.1)$ & 59 (52.2) & $11(9.7)$ \\
\hline & October & 1719 & $140(8.1)$ & $67(47.9)$ & $63(45.0)$ & $10(7.1)$ \\
\hline & November & 1288 & $120(9.3)$ & $51(42.1)$ & $58(47.9)$ & $12(9.9)$ \\
\hline
\end{tabular}




\section{Discussion}

Malaria is a public health problem in terms of morbidity and burden on health care facilities, accounting for the increasing percentage of outpatients in most health facilities in different regions in Ethiopia (4). The present study revealed that the average annual malaria prevalence was 7.7\% (95\% $\mathrm{Cl} ; 7.3-8.1)$. This finding was lower than the study conducted elsewhere in Kola Diba, North Gondar, Northwest Ethiopia (4), Adi Arkay, North Gondar, Northwest Ethiopia (12), Abeshge, south-central Ethiopia (6), Dembecha Health Center, West Gojjam Zone, Northwest Ethiopia (8) and Woreta Health Center, Northwest Ethiopia (13), and higher than other study findings conducted in of Felegehiwot referral Hospital catchment areas, Bahir Dar, northwest-Ethiopia Ethiopia(7) and Halaba special district, Southern Ethiopia (14). The difference might be due to variations in malaria diagnosis techniques and the skills of the laboratory personnel to detect and identify malaria parasites. Moreover, the implementation of malaria prevention and control activities might differ from one area to another. Besides, there might be demographic (sex, age) and geographic (attitude, temperature, rain fall) and economical activities differences that also had an effect on the prevalence of malaria.

The average annual trend of malaria revealed that there was slight increment malaria prevalence in the first two years (2015 and 2016$)$ compare to the year of 2014 , but statistically it was insignificant. However, in the last three years $(2017,2018$ and 2019) the trend showed that significant reduction of malaria prevalence. The prevalence was reduced by $68 \%, 60 \%$ and $69 \%$ in the year of 2017,2018 and 2019 , respectively. The possible reasons for malaria reduction during this period (2017-2019) might be due to the increased attention to malaria control and preventive activities by different responsible bodies, increased awareness of the community on use of ITNs, insecticide spraying, drainage system of mosquito breeding sites and climate change at national and international level. Integrated control strategies are underway in the local area as part of the nationwide malaria control activities (15). However, the observed prevalence in this study is still considerable.

This study demonstrated that in average of the six years, P. vivax was the predominant species, although there was a species fluctuation from year to year. The proportion of P.vivax, P. falciparum and mixed infections was $47.2 \%, 45.6 \%$, and $7.2 \%$, respectively. This finding was consistent with the study conducted in Adama City, East Shoa Zone, Oromia, Ethiopia (15), Halaba health center Southern Ethiopia (14). The predominance of P. vivax might be due to relapse of dormant liver stages or increased treatment pressure against P. falciparum (16)and Southwest Ethiopia, around Gilgel gibe dam and 10 kilo Metter far from Gilgel gibe dam (3). However, this finding was disagreement with the study conducted at two health centers Gorgora and Chuahit in Dembia district (17), catchment areas of Felegehiwot referral Hospital (7) and Kola Diba, North Gondar, Northwest Ethiopia (4) which reported that P. falciparum was the predominant species. Moreover, the trend of P.vivax showed reduction whereas, P. falciparum showed increment trend. In the last three year of the six year periods, P. falciparum had become the predominant plasmodium species. The fluctuated proportion of plasmodium species might be attributed by heterogeneous parasite species and disease distribution include differences in genetic polymorphisms underlying parasite drug resistance and host susceptibility, mosquito vector ecology and transmission seasonality. Plasmodium species interact might have geographical differences and these interactions may even change from year to year in a given locale (18). The finding also revealed that there was fluctuated increment in the proportion of mixed infection.

The prevalence of malaria was varied among different seasons ranging from $6.6-8.8 \%$, and these variation was statistically significant. The highest peak was observed in autumn (8.8\%) and the lowest peak was observed in the winter season (6.6\%). The malaria prevalence was reduced by $16 \%$ in the winter. However, where the sex and age were adjusted, the peak prevalence was observed in summer rather than autumn, in which the prevalence was increased by $32 \%$. The reason might be due to climate change from year to year. In Ethiopia, summer is the season when the heavy rain fall is observed and it is not favorable season for vector spreading (15). However, there is rain fall variation from year to year (19). Changes in temperature, rainfall, and relative humidity due to climate change are estimated to influence malaria directly by modifying the behavior and geographical distribution of malaria vectors and by changing the length of the life cycle of the parasite. Climate change is also expected to affect malaria indirectly by changing ecological relationships that are important to the organisms involved in malaria transmission (the vector, parasite, and host) (20).

The current study revealed that males were more affected by malaria infection than females. The odds of malaria positivity rate among males were 1.41 times higher than females. The reason behind the high malaria cases in male might be due to the facts that males are involved in outdoor activities and are mobile to malaria-endemic areas seeking temporary employment, whereas females do not perform field activities rather they are cookers and stay at home which might reduce the risk of infection.

Age was also contributing factor for the prevalence of malaria. It was higher in younger age groups than the older age groups. The odds of malaria positivity rate among less than five years old children and 5-14 years old were 1.60 and 1.64 times higher than age group of greater than 55 year old, respectively. The reason might be these age groups may be less immune commutate than the older age groups ( $>55$ year old). This was supported by the world health organization report (21). The study also showed that the odds of malaria positivity rate among early working group (15-24) and primarily working groups (25-54) were, 2.45 and 1.82 times higher than age group of greater than 55 year old, respectively. The reason behind the high malaria cases in the mentioned age group of 15-24 and 25-54 years old might be the former one, that is males in this age group are involved in outdoor activities and are mobile to malariaendemic areas seeking temporary employment, whereas the older age group do not perform field activities rather they are stay at home which might reduce the risk of infection.

The findings of this study might suffer from the fact that it is secondary data based on records; the reliability of the recorded data couldn't be ascertained. Moreover, the collected data relayed on the laboratory logbook which lacks participants' body temperature, clinical presentations and residence. It also lacks the information regarding to the weather conditions of the month, seasons and years.

\section{Conclusion}


There has been a significant declining trend of the overall positivity rate of malaria in the study area. However, it is still a major public health problem and requires extra efforts for further reduction. In average, the highest peak of malaria cases in was observed during the autumn seasons. However, there was high fluctuation from year to year. Moreover, males, under five children and the younger age groups were more affected compare to the older age groups. In addition, even P. vivax was the predominant plasmodium species in the allover trend, there was a high fluctuation of plasmodium species from year to year and season to season. Therefore, control activities should be continued and strengthened manner in the study area considering these variability.

\section{Abbreviations}

AOR: Adjusted Odds Ratio; Cl: Confidence interval; P. falciparum: Plasmodium Falciparum; P.vivax: Plasmodium Vivax

\section{Declarations}

\section{Authors' contributions:}

$\mathrm{AM}, \mathrm{AA}, \mathrm{AE}$ and $\mathrm{BA}$ participated in the study design, undertook the data collection, analyzed the data. $\mathrm{YG}$ analyzed the data, wrote the manuscript and participated on the revision of the manuscript. MB participated in the study design, analyzed the data and on the revision of the manuscript. All authors have read the manuscript and approved it to submit for publication.

\section{Acknowledgements:}

We are grateful to University of Gondar specialized referral hospital managers and laboratory personnel.

\section{Competing interests:}

The authors declare that they have no competing interests.

\section{Availability of data and materials:}

All data generated or analyzed during this study are included in this published article

\section{Consent for publication:}

Not applicable in this section.

\section{Ethics approval and consent to participate:}

Ethical clearance was obtained from the School of Biomedical and Laboratory Sciences, College of Medicine and Health Science, University of Gondar. After discussing the purpose and method of the study, verbal consent was sought from the Medical director of University of Gondar Specialized Referral Hospital before the data collection

Funding

No fund

\section{References}

1. Kassa A, Beyene BBJSJoPH. Climate variability and malaria transmission-fogera district. Ethiopia, 2003-2011. 2014;2(3):234-7.

2. Ayele DG, Zewotir TT, Mwambi HG. Prevalence and risk factors of malaria in Ethiopia. Malar J. 2012;11:195.

3. Sena LD, Deressa WA, Ali AA. Analysis of trend of malaria prevalence in south-west Ethiopia: a retrospective comparative study. Malar J. 2014;13:188.

4. Alemu A, Muluye D, Mihret M, Adugna M, Gebeyaw M. Ten year trend analysis of malaria prevalence in Kola Diba, North Gondar, Northwest Ethiopia. Parasites vectors. 2012;5:173.

5. Tesfa H, Bayih AG, Zeleke AJ. A 17-year trend analysis of malaria at Adi Arkay, north Gondar zone, Northwest Ethiopia. Malar J. 2018;17(1):155.

6. Yimer F, Animut A, Erko B, Mamo H. Past five-year trend, current prevalence and household knowledge, attitude and practice of malaria in Abeshge, southcentral Ethiopia. Malar J. 2015;14:230.

7. Yimer M, Hailu T, Mulu W, Abera B, Ayalew W. A 5 year trend analysis of malaria prevalence with in the catchment areas of Felegehiwot referral Hospital, Bahir Dar city, northwest-Ethiopia: a retrospective study. BMC Res Notes. 2017;10(1):239.

8. Haile D, Ferede A, Kassie B, Abebaw A, Million Y. Five-Year Trend Analysis of Malaria Prevalence in Dembecha Health Center, West Gojjam Zone, Northwest Ethiopia: A Retrospective Study. Journal of Parasitology Research. 2020;2020:8828670.

9. Federal Democratic Republic of Ethiopia Ministry of Health, National Malaria Guidelines, fourth edition, Federal Democratic Republic of Ethiopia Ministry of Health, Availabel from: 
https://www.humanitarianresponse.info/sites/www.humanitarianresponse.info/files/documents/files/eth_national_malaria_guidline_4th_edition.pdf:Acct date June 1,2021.

10. United State Agency International Development,PRESIDENT'S MALARIA INITIATIVE ETHIOPIA. Malaria Operational Plan FY. 2018, USA PRESIDENT'S MALARIA INITIATIVE collaboration with Department of Health and Human Science, Center for Disease controll and prevention, United State of America Department of State, Available from; https://www.pmi.gov/docs/default-source/default-document-library/malaria-operational-plans/fy-2018/fy-2018ethiopia-malaria-operational-plan.pdf?sfvrsn=5, Access date June 1, 2021.

11. Levin KAJE-bd. Study design III: Cross-sectional studies. 2006;7(1):24.

12. Tesfa H, Bayih AG, Zeleke, AJJMj. A 17-year trend analysis of malaria at Adi Arkay, north Gondar zone. Northwest Ethiopia. 2018;17(1):155.

13. Derbie A, Alemu M. Five Years Malaria Trend Analysis in Woreta Health Center, Northwest Ethiopia. Ethiopian journal of health sciences. 2017;27(5):46572.

14. Shamebo T, Petros B. Trend analysis of malaria prevalence in Halaba special district, Southern Ethiopia. BMC Res Notes. 2019;12(1):190.

15. File T, Dinka H, Golassa L. A retrospective analysis on the transmission of Plasmodium falciparum and Plasmodium vivax: the case of Adama City, East Shoa Zone, Oromia, Ethiopia. Malaria Journal. 2019;18(1):193.

16. Dedgeba S. HM. Malaria trends in Silt'i district from 2009-2015 and current childhood malaria in K'ibbet hospital, south-central Ethiopia MalariaWorld. Journal. 2017;8(22):1-5.

17. Addisu A, Tegegne Y, Mihiret Y, Setegn A, Zeleke AJ. A 7-Year Trend of Malaria at Primary Health Facilities in Northwest Ethiopia. Journal of Parasitology Research. 2020;2020:4204987.

18. Zimmerman PA, Mehlotra RK, Kasehagen LJ, Kazura JW. Why do we need to know more about mixed Plasmodium species infections in humans? Trends Parasitol. 2004;20(9):440-7.

19. Simane B, Beyene H, Deressa W, Kumie A, Berhane K, Samet J. Review of Climate Change and Health in Ethiopia: Status and Gap Analysis. The Ethiopian journal of health development = Ya'Ityopya tena lemat mashet. 2016;30(1 Spec Iss):28-41.

20. Alemu A, Abebe G, Tsegaye W, Golassa L. Climatic variables and malaria transmission dynamics in Jimma town, South West Ethiopia. Parasites vectors. 2011;4(1):30.

21. WHO, malaria fact sheet report; World Health Organization. Available from https://www.who.int/news-room/fact-sheets/detail/malaria, Access date June $18,2021$.

\section{Figures}

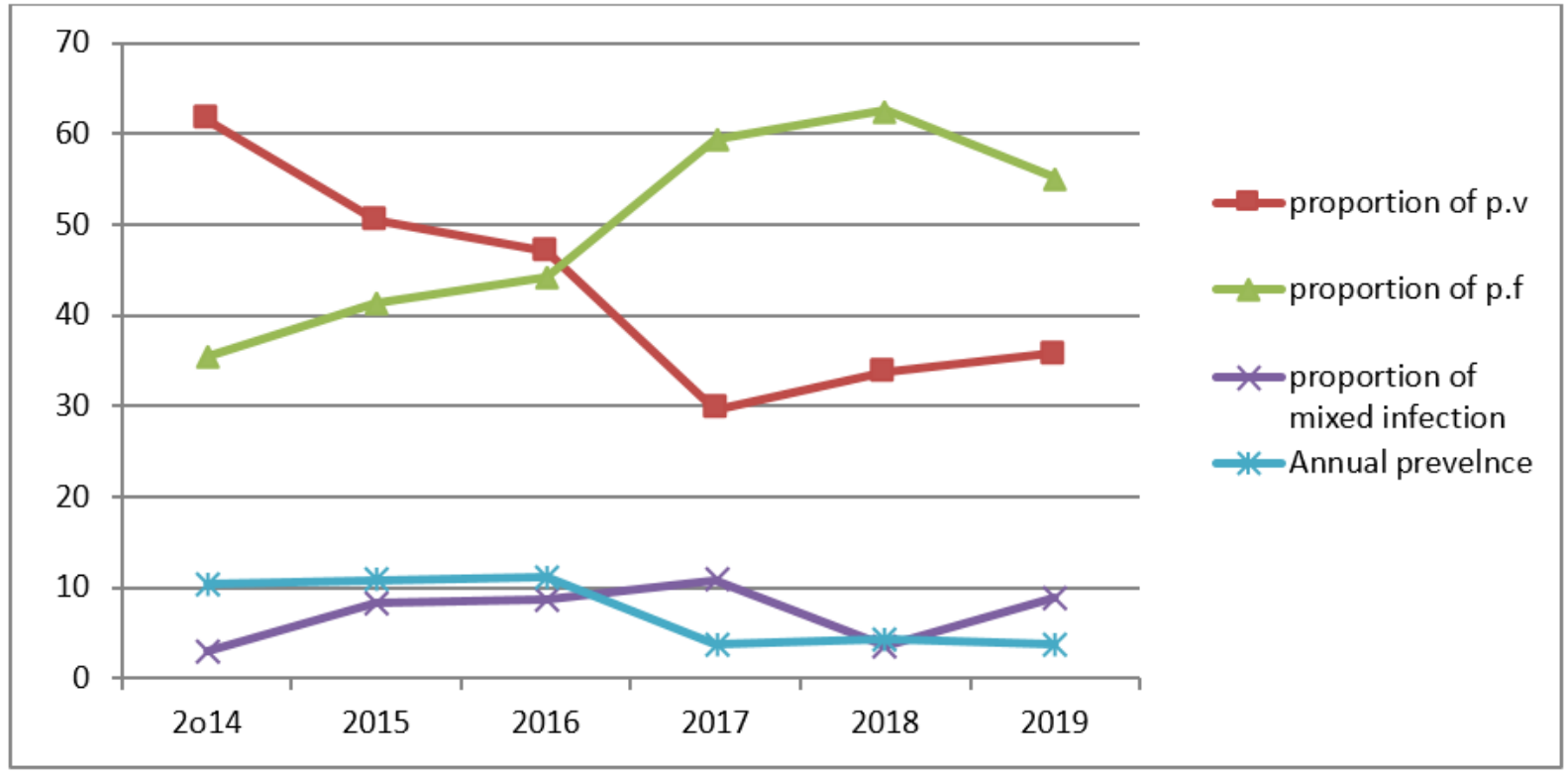

Figure 1

Annual trend of malaria prevalence and proportion of plasmodium species at University of Gondar specialized referral hospital from 2014-2019 P.f: plasmodium falciparum; P.v: plasmodium vivax 


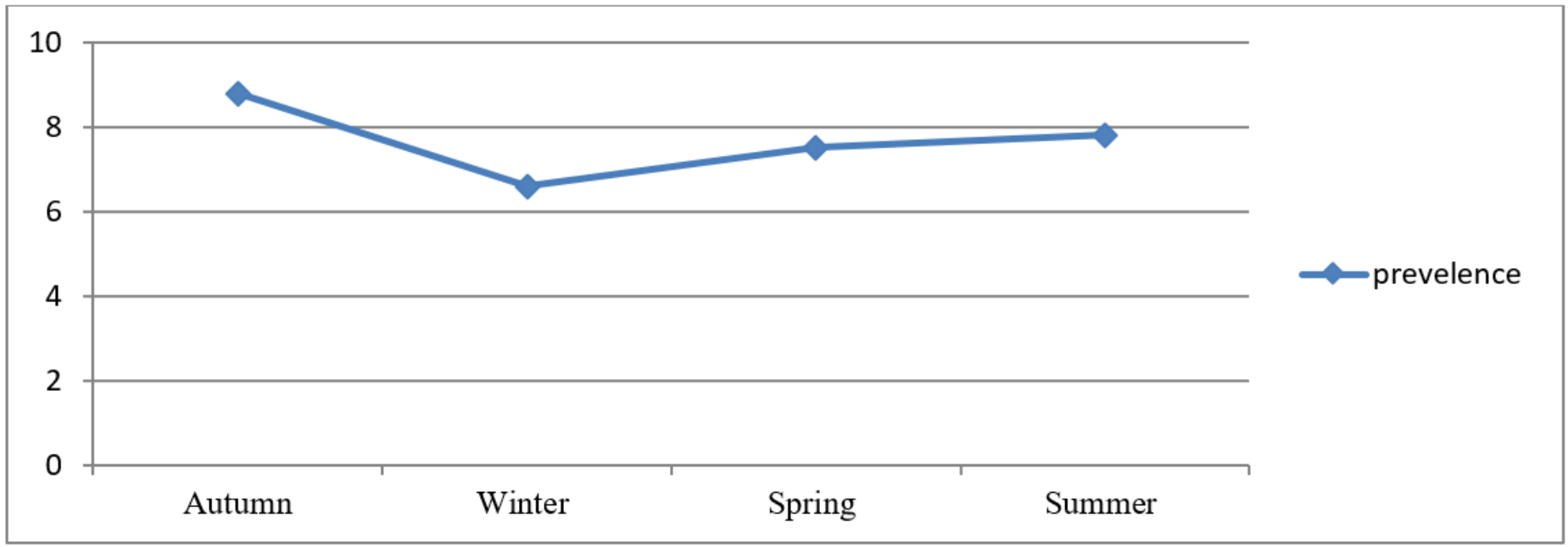

Figure 2

Seasonal variations of malaria prevalence among blood smear microscopy at University of Gondar Specialized Referral Hospital from 2014 to 2019

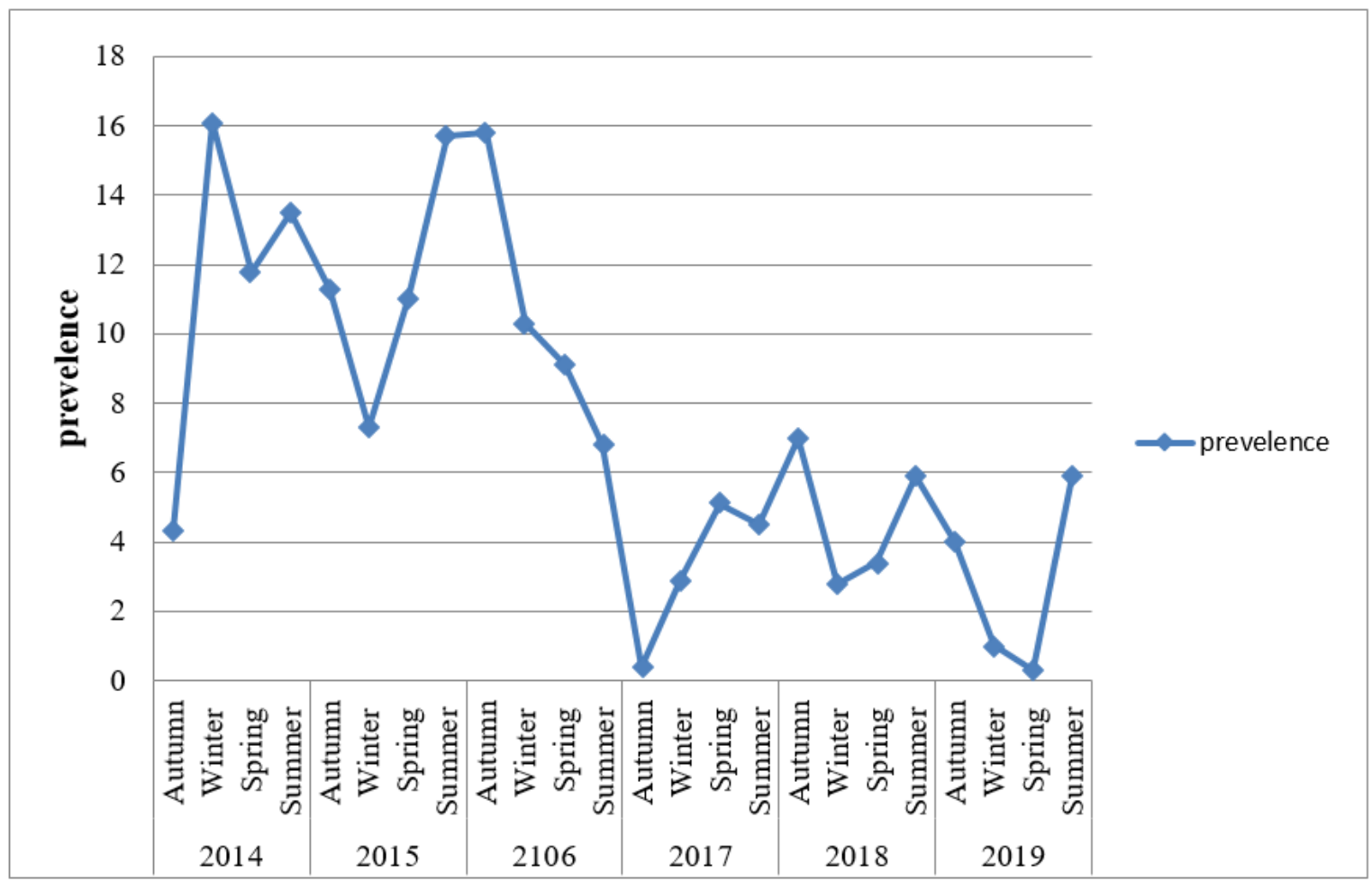

\section{Figure 3}

Seasonal variations of malaria prevalence in each year among patients requested for malaria examination at University of Gondar Specialized Referral Hospital from 2014 to 2019 